\title{
Reply to Letter to the Editor: Re: The Role of Gender in Careers in Medicine: a Systematic Review and Thematic Synthesis of Qualitative Literature
}

Dr. Salib points out an important issue hidden within our use of terms like "impostor syndrome" and "burnout." Both assume that these cultural norms are appropriate expectations for physicians. Just as we should recognize the structural problems in our workplace if healthcare workers are suffering burnout from chronic work-related stress, so too should we recognize that our cultural norms espouse rigid, "masculine" archetypes. Just as the problems with physician burnout will not be fixed if we exclusively focus on building resilience, so too can the challenges of gender inclusivity not be fixed by individual adaptation to these outdated norms. Instead, professional norms should align with professional expectations and the value of individual contributions. Women will not reach their full potential exclusively through developmental programs focused on adopting stereotypically "masculine" behaviors. If we are to build a culture of learning and practice in medicine where diverse individuals can thrive, we will need to recognize the flaws in our unexamined expectations of our work environment.

On behalf of my co-authors, Beatrice Telzak, Jacquelyn Shaw, Calder Hollond, Juliana Magro, Joseph Nicholson, and Gwendolyn Quinn,

Sincerely,

Abigail Ford Winkel.

Received: 4 October 2021

Accepted: 29 October 2021

Published online January 17, 2022
$J$ Gen Intern Med 37(4):973

DOI: $10.1007 / \mathrm{s} 11606-021-07259-6$

(c) Society of General Internal Medicine 2021

\begin{abstract}
Abigail Ford Winkel, $M D, M H P E^{1}$
${ }^{1}$ Department of Obstetrics and Gynecology, Institute for Innovations in Medical Education, New York University Grossman School of Medicine New York, NY, USA
\end{abstract}

Corresponding Author: Abigail Ford Winkel, MD, MHPE; Department of Obstetrics and Gynecology, Institute for Innovations in Medical Education, New York University Grossman School of Medicine, New York, NY, USA (email: abigail.winkel@nyumc.org)

Publisher's Note Springer Nature remains neutral with regard to jurisdictional claims in published maps and institutional affiliations. 